\title{
eJRIEPS
}

Ejournal de la recherche sur l'intervention en éducation physique et sport

49 | 2021

Varia

\section{Un corps d'élève décerné par un professeur ; une étude de cas sur le « stade du miroir » en EPS}

A student body awarded by a teacher; a clinical case study on something from the "mirror stage" in P.E.

Yves-Félix Montagne

\section{(2) OpenEdition}

\section{Journals}

Édition électronique

URL : http://journals.openedition.org/ejrieps/6006

DOI : $10.4000 /$ ejrieps.6006

ISSN : 2105-0821

Éditeur

ELLIADD

Référence électronique

Yves-Félix Montagne, «Un corps d'élève décerné par un professeur ; une étude de cas sur le «stade du miroir » en EPS », eJRIEPS [En ligne], 49 | 2021, mis en ligne le 01 avril 2021, consulté le 08 avril 2021. URL : http://journals.openedition.org/ejrieps/6006 ; DOI : https://doi.org/10.4000/ejrieps.6006

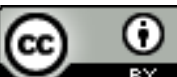

La revue eJRIEPS est mise à disposition selon les termes de la Creative Commons Attribution 4.0 International License. 


\section{Un corps d'élève décerné par un professeur ;} une étude de cas sur le « stade du miroir » en EPS.

Yves-Félix Montagne*

* INSPE Besançon, Laboratoire ELLIADD (EA 4661), Université de Franche Comté

Résumé

Cette étude de cas montre, depuis le champ conceptuel et méthodologique de la clinique psychanalytique, que, en EPS, la parole d'un élève n'est pas que verbalisation ou communication. Elle montre aussi que des gestes professoraux permettant aux élèves d'améliorer l'accès à leur corps peuvent s'incarner par de simples hochements de tête et par le fait de "répondre pas vraiment" aux demandes d'une élève. Le savoir-y-faire langagier de Mme $D$, enseignante d'EPS, fait ainsi écho aux demandes surprenantes d'Assia, élève de $4^{e}$ dans l'APSA combat : "Madame, comment je peux savoir comment je suis? Dites-moi si je suis bien!". Une observation clinique et l'analyse interprétative d'entretiens individuels non directifs permettent de penser que la relation langagière et visuelle, qui unit enseignante et élève dans le cas présenté, renvoie au transfert freudien et à quelque chose $d u$ "Stade du Miroir ", moment du développement humain que la psychanalyse définit comme celui où regard et parole de l'adulte décernent à l'enfant l'accès à son corps. Ce postulat permet d'envisager un rôle autre pour l'EPS et pour l'action professorale en EPS et engage à se demander comment transmettre cela en formation. Mots clés : EPS, corps, psychanalyse, stade du miroir, parole, transfert

A student body awarded by a teacher; a clinical case study on something from the "mirror stage" in P.E.

Summary

This case study shows, from the conceptual and methodological field of psychoanalytic clinic, that, in PSE, a student's speech is not only verbalization or communication and that effective teaching gestures (allowing students to improve access to their bodies) can be embodied by nodding their heads and "not really responding" to a student's requests. The language "know-how » of Mrs D, a P.E.teacher, echoes the surprising request of Assia, a 8th grade student in a wrestling lesson : "Ma'am, how can I know how I am?" Tell me if I am okay! ". A clinical observation and the interpretative analysis of non-directive individual 


\section{eJRIEPS 49 avril 2021}

interviews allow to think that the linguistic and visual relationship, which unites teacher and student in this case, refers to the Freudian transference and to something of the Mirror Stage ; time of human development where, according to psychoanalysis, the adult's eyes and words provide access to the child's body. This assumption makes it possible to envisage a different role for P.E. and for teaching action in P.E. and involves us asking how to transmit this in teacher training.

Key words: physical education, body, psychoanalysis, mirror stage, speech, transference

« Le corps, c'est le langage qui le décerne »

Lacan, J. (2001). Radiophonie. In Autres Écrits.

Paris : Seuil, p. 409.

\section{Introduction}

II s'agit dans ce travail, orienté par les écrits de Freud et l'enseignement de Lacan, d'une tentative d'analyse de l'interrelation de parole entre professeur et élève en Éducation Physique et Sportive (EPS) et plus particulièrement du rôle de cette relation dans les bénéfices qu'une élève adolescente peut trouver dans l'accès à son corps, au-delà des compétences cognitive-motrices et méthodologique développées habituellement en EPS. On entendra ici l'accès à son corps comme le fait pour un être humain, de prendre connaissance et conscience de son corps et d'en avoir (à minima) le contrôle moteur.

Cette perspective repose sur la prise en compte de la singularité des liens subjectifs existant entre un enseignant et un élève en EPS. La spécificité corporelle de cette discipline scolaire, au-delà de la motricité, repose sur le fait que dans les gymnases le dire induit le faire et que ce sont les élèves et leurs corps, observés et parlés, qui sont l'objet d'enseignement. En mathématiques, le professeur regarde et commente la copie, le tableau. En langues vivantes, le professeur entend le vocabulaire, la rectitude ou l'erreur dans la grammaire et la prononciation, il s'attache à l'énoncé moins à l'énonciation. En arts plastique il commente les productions artistiques et attend et entend les ressentis affectifs provoquées chez les élèves par « les œuvres ». En fait dans toutes les disciplines « en classe », les paroles des professeurs organisent les corps qui ne sont pris que comme des supports aux apprentissages intellectuels des élèves. En EPS, la parole de l'enseignant crée, supporte et ordonne des apprentissages dont les corps sont l'objet et l'objectif, et la parole des élèves exprime des ressentis sur les effets des corps mis en jeu autant que des commentaires sur des actions ou des gestes. Ces « dits » organisent et structurent la classe et les contenus 


\section{eJRIEPS 49 avril 2021}

d'enseignement. Le lien éducatif se tisse autour du paradoxe de paroles qui montrent et laissent entendre et de regards qui renseignent ou demandent.

Une telle lecture de la relation pédagogique pose que la voix et le regard de l'être humain ne sont pas uniquement liés à la vision et à l'audition et Moll (2003) a montré les effets du regard et de la parole du professeur en classe. Ces travaux reposent sur le fait que Freud (1915) a établi que l'acte de regarder pris comme une pulsion scopique intéresse l'œil et le regard autrement que comme organe optique. La pulsion scopique, désigne la tendance à rechercher la satisfaction apportée par le regard (regarder, être regardé, se faire regarder). Elle peut pour un sujet permettre un contentement plus ou moins explicite et conscient. Dans cette organisation psychique, se joue une relation à l'autre comme objet de jouissance (au sens lacanien de satisfaction pas forcément agréable et en cela différente du plaisir). Dans la même logique, l'acte de parler, pris comme pulsion invocante (qui appelle) n'est pas seulement la mobilisation de la voix pour communiquer. La pulsion invocante désigne la tendance à rechercher la satisfaction apportée par le fait de demander, d'être demandé ou de se faire demander.

N'existerait-il pas alors, dans l'outillage professoral, une compétence insue (et peu abordée en formation) ; savoir-y-faire avec l'écoute, le regard et la parole?

Ce travail supplémente une étude de cas réalisée sur le même thème (l'usage qu'un élève fait de la parole et du regard professoral) avec un élève de 4 ans (Montagne, 2014) qui, durant un cours d'EPS de moyenne section de maternelle, sollicitait sa professeure des écoles pour mieux comprendre l'étrange que lui faisait vivre son corps engagé dans la pratique du Roller en EPS.

Cette étude de cas avait établi que les questions d'un élève envers sa professeure pouvaient se situer au-delà d'une demande de conseils techniques pour " bien patiner ॥ et/ou éviter de tomber et de se faire mal. La parole et le regard d'un autre essentiel (un professeur), et l'usage qu'en fait un sujet (un élève), ont ainsi été caractérisés comme des moyens qu'un enfant peut convoquer pour affronter des moments d'angoisse liée à des perturbations du rapport au corps contextuelles (liée à la pratique d'Activité Physique Sportive et Artistique (APSA) et améliore sa «selbstbewusst» (connaissance/compréhension de soi) au-delà de l'accès à son corps.

La question de recherche posée ici sera donc double :

- Quelles sont les causes psychiques et la nature des paroles d'une adolescente de $4^{\mathrm{e}}$ en EPS, Assia (prénom d'emprunt), qui pose des questions inhabituelles à Mme D, sa professeure d'EPS (dans l'APSA combat) : «Madame, ma jambe, 


\section{eJRIEPS 49 avril 2021}

dites-moi vous ! Comment je peux savoir comment je suis ? Dites-moi si je suis bien!»?

- Quelle est la nature de la réponse de Mme D, qui dit « répondre, mais pas vraiment » aux questions de son élève?

Après avoir précisé le cadre théorique et méthodologique de cette recherche, le cas Assia sera présenté. La réponse de Mme D sera également analysée et expliquée. Seront enfin évoquées des perspectives d'usage des conclusions présentées pour la formation des enseignants.

\section{Le cadre épistémologique de la psychanalyse.}

\subsection{L'inconscient et l'être humain divisé}

La psychanalyse soutient qu'il existe en l'homme la réalité matérielle consciente (Wirklichkeit) et la réalité psychique inconsciente (Realität). Cet état fait de l'être humain un sujet divisé, car déterminé en partie dans ses paroles et ses actes par des causes et des buts dont il n'a pas connaissance. Le mot "sujet » utilisé par Lacan pour désigner l'être humain parlant n'est ni le sujet de la conscience, celui de l'énoncé (le je dont on parle) ou de l'énonciation (le je qui parle) de la linguistique. Le sujet est l'être humain en tant qu'effet de signifiant (au sens saussurien du terme), c'est-à-dire en tant que conséquence de sa parole, sur l'autre.

Ainsi, puisque « la psychanalyse a développé une éthique de l'altérité et de la singularité » qui engage « une réflexion constante sur la bonne distance avec un autre » (Cifali, 2002, p. 164), elle apparaît comme un prisme intéressant pour comprendre une part de ce qui se joue dans la relation pédagogique en classe. De la sorte il est possible de concevoir que l'espace-temps de la leçon partagé par élèves et professeur n'a pas la même valeur subjective pour tous et que les relations éducatives, de fait, n'ont que des significations singulières. Surtout quand ce qui se joue en classe apparaît illogique ou incompréhensible, difficile ou impossible. La psychanalyse traite en effet « les embrouilles de l'être parlant [...] et l'énigme du désir quand il provoque un dis-corps, cette discorde du corps et de l'être " (Bonnaud, 2015, p. 21).

Postic (1979, p. 224) affirme dans cet esprit que « la psychanalyse offre la possibilité d'interroger ce qui se passe dans le champ pédagogique et engage l'éducateur à se découvrir dans son rapport à l'élève " La psychanalyse permet donc d'éclairer le champ pédagogique et de s'y orienter autrement. Bien préciser ici que, si Freud a exposé la 


\section{eJRIEPS 49 avril 2021}

psychanalyse à la fois comme une pratique thérapeutique de parole pour soigner et comme un corpus de connaissances nommant les phénomènes repérés, « elle ne préconise pas le conseil ou le compromis. Elle soulève le désir, ce qui donne une autre façon de se voir, et un autre regard sur les autres » (Bonnaud, 2013, p. 163).

De nombreux chercheurs Filloux (1974), Cifali (1994), Blanchard Laville (2001), s'attachent donc à analyser et comprendre le champ de l'éducation depuis cette épistémologie du désir en jeu dans les classes. Les STAPS et l'EPS aussi, à travers les travaux de Pujade-Renaud (1983) Labridy (1997), Terrisse (1997), Lévêque (1985), Carnus (2010), Buznic- -Bourgeacq \& Terrisse (2013), offrent cette lecture particulière du lien éducatif et des enjeux de l'enseignement dans le gymnase.

Toutes ces approches reposent sur une conception de l'être humain (et donc des élèves et du professeur) divisé telle que forgée par Freud (1923). Divisé entre le Ça, le Moi, et le surmoi, trois instances psychiques internes et antagonistes qui sont respectivement le lieu ;

- Des pulsions, où le désir s'exprime sans barrière éthique ou sociale en cherchant à éviter le déplaisir et satisfaire le sujet

- De l'image et la perception consciente qu'un être humain a de lui-même

- De l'intériorisation des règles, des interdits fondamentaux, de la loi et de la morale qui poussent et contraignent toujours plus.

On peut alors se demander en quoi les questions d'Assia témoignent d'une structuration particulière de ces trois instances, et quelle instance supporte les réponses de Mme D? En enrichissant les propos freudiens des apports de la linguistique et du structuralisme, Lacan a désigné l'être humain comme un Sujet divisé en trois registres psychiques noués entre eux à la manière d'un nœud borroméen (nouage dans lequel si un des anneaux se rompt l'ensemble se défait) : le Réel, le Symbolique et l'Imaginaire.

- Le Réel représente la partie inatteignable du sujet, « quelque chose qui est à la limite de notre expérience » (Lacan, 1975, leçon XI, 8 février 1956), soit le nonidentifiable de la vie, ni par des mots, ni par des images.

- L'Imaginaire est ce qui désigne les représentations du monde que se fabriquent le sujet, les significations (identification, images) qu'il se donne de lui et de la réalité du monde.

- Le Symbolique définit la partie du sujet organisée par le langage, et détermine les liens entre le sujet et les autres, à travers ce qu'il émet et ce qu'il reçoit par la parole. 


\section{eJRIEPS 49 avril 2021}

Dès lors, on peut concevoir que professeur comme élève(s) sont divisés entre ce qu'ils éprouvent (du côté du Réel), disent (du côté du Symbolique), se représentent (du côté de l'Imaginaire) et que leur(s) interrelation(s) éducative(s) sont colorisées (comme on dit d'un film noir et blanc) par ces registres psychiques.

En EPS, en sus de ces considérations, le corps des élèves est mis en jeu de façon incontournable, et en soi comme en tant que média de communication entre deux sujets, il peut être pensé lui aussi comme divisé. On peut ainsi, en s'appuyant sur les travaux de Sami Ali (1990) identifier un corps Réel (senti, vécu, l'organisme), un corps symbolique (parlé et parlant, l'idée du corps) et un corps imaginaire (représenté, imaginé, l'image du corps).

II semble alors important de s'attacher à savoir sur quel corps Assia demande des « feedback » quand elle demande à sa professeur «Madame, comment je peux savoir si je suis comme il faut? » De même, on peut se demander dans quel registre se situent les réponses de Mme D : «Assia, je l'entends, je la regarde, mais je ne sais pas si je lui réponds. En vérité, je ne réponds vraiment »? (pour tout ce texte, les paroles d'Assia et de Mme D sont écrites telles que prononcées et convoquées « entre guillemets et en italique »).

Au-delà de la pluralité intime de l'homme, la psychanalyse fait aussi choix de le poser comme sujet désirant et plus particulièrement comme désirant obtenir de l'autre des éléments de réponse sur ce qu'il est (pour lui, mais aussi pour l'autre). La parole est alors à la fois carburant et comburant de cette demande.

\section{2 la parole équivoque et invocante}

La parole dit Soler (2019, p. 23) « est constitutive de l'humain avec la différence de ce qui vient du corps, à savoir son image et la pulsion ». Fort du fait que le Sujet pour la psychanalyse est effet de parole, Soler ajoute qu'il est un résultat de « l'inter signifiance », soit de la relation de parole entre deux êtres qui parlent «à » (un interlocuteur dans la réalité ou dans l'imaginaire) et parlent « pour» (assouvir quelque chose de leur désir) avec toute la subjectivité et l'équivoque que cela comporte. Bien évidemment l'école (et donc l'EPS incluse) est baignée dans ce lien discursif.

L'équivoque consécutive à tout échange langagier peut être due à des confusions liées à l'homophonie (la similitude du son peut changer le sens de ce qui est dit/entendu. Par exemple : je c'est pas moi/je sais pas moi) ou aux effets de l'écart entre le sens dénotatif d'un mot (dans lequel sa valeur a une signification univoque) et son sens connotatif (que lui attribue celui qui l'écoute ou que donne le contexte d'énonciation). Ainsi le sens latent 


\section{eJRIEPS 49 avril 2021}

(Freud, 1895) d'une parole (ce qu'elle peut vouloir dire) est différent de son sens manifeste (ce qu'elle dit) fonde l'écart entre dit et dire, ou entre énoncé et énonciation.

L'énoncé correspond à ce qui est dit quand un sujet parle. C'est le résultat linguistique (désignation et identification) d'une parole prononcée; c'est « le dit ». L'énonciation est l'acte d'énoncer, mais c'est aussi « le dire » de la parole, c'est-à-dire ce qui s'entend et se transmet en filigrane dans ce qui est prononcé. Arrivé (2003, p. 18) souligne que « le choix d'une énonciation dans le système grammatical baigne en grande partie dans l'inconscient ». La psychanalyse soutient que dans ce choix non décidé consciemment se révèle une part du désir de celui qui parle.

Ainsi, la dualité entre ce qui est dit et voulait être dit, ou entre ce qui se dit et ce qui s'entend, dont témoigne l'expression familière « je n'ai pas voulu dire ça » quand deux interlocuteurs notent un désaccord conversationnel, fait que la question d'un sujet (élève) à un autre (professeur) peut vouloir dire autre chose que ce qui est réclamé explicitement. De fait, quand un sujet s'adresse à un autre dans une forme interrogative, il pose une question (un dit) qui peut (ou pas) avoir valeur de demande (un dire). Mais toute question, en tant qu'énoncé n'est pas une demande, car la psychanalyse enseigne que toute parole n'est pas un dire.

La valeur signifiante de la demande, comme invocation (in-vocare, en latin signifie mettre en voix quelque chose de son désir en s'adressant à l'autre), repose sur une relation dissymétrique entre deux sujets et s'explique par l'analyse du cri du nourrisson (l'infans, celui qui ne parle pas). Encore incapable de paroles, en proie à un manque organique (la faim par exemple), il crie vers sa mère, qui répond bien au-delà de ce que ces cris signifient. À partir des trois expressions grecques désignant la voix, Bentata (2011) définit ainsi le lien langagier entre mère et enfant:

- Phtoggos, le cri, que l'on peut assimiler à l'appel à la présence impérative de l'autre, la demande du nourrisson ;

- Op's, la parole rassurante, c'est-à-dire les modulations de la prosodie et de ton de voix spécifiquement destinées à apaiser celui qui s'exprime en attestant d'une présence et d'une écoute ;

- Aïode, qui est le contenu de la parole, le savoir transmis par les mots qui renseigne le sujet sur ce qu'il demande, sur son état, sur l'état du monde.

Se dessine alors pour expliquer les relations premières entre mère et enfant le scénario suivant : l'enfant crie (Phtoggos), la mère répond pour rassurer et demande : « Mais oui mon bébé, je t'entends! Que veux-tu? » (Op's), puis elle répond à sa propre question en 


\section{eJRIEPS 49 avril 2021}

interprétant les signes émis par le bébé et nomme par ses mots le ressenti qui a provoqué le cri : « Tu as faim ! (Aïode).

À la lumière de ces éléments, ne pourrait-on pas alors envisager de considérer autrement les questions d'Assia? II est par exemple possible de les penser, si l'on pose comme postulat que le lien éducatif rejoue quelque chose du lien parental (notamment au niveau de la pulsion orale (nourriture/savoir)), comme des appels à Mme D depuis le registre de Phtoggos, attendant autant Op's (le retour rassurant) que Aïode (le savoir promis par l'EPS) ?

\section{La méthode clinique analytique.}

\subsection{La clinique}

La clinique mise en œuvre dans ce travail est une méthodologie de recherche qualitative qui recueille et analyse des données (ce mot étant à relativiser puisque le corpus des paroles d'un sujet qui sert de matériel clinique n'est pas « donné », mais issu d'une construction interlocutoire, rencontre entre la parole singulière d'un sujet et l'interprétation subjective du chercheur) et produit des hypothèses interprétatives plausibles sous forme d'étude de cas et non sur une démonstration.

II s'agit dans cette démarche de s'intéresser au point de vue d'un sujet singulier inclus dans le lien social en lui donnant la parole. II s'agit ici d'Assia, élève de 4e, dans son cours d'EPS, en relation avec sa professeure, en écho à ce qu'Assia vit dans sa famille. Dans cette considération propre à ce que Lagache (1949) appelait l'homme en situation, c'est le télescopage entre quelque que chose d'entendu/vu et la rupture du sens commun entre ce qui est remarqué et ce qu'on pouvait s'attendre à constater dans cette situation, qui alerte le chercheur. C'est le fait de se dire « pourquoi ce sujet n'est pas où je/on l'attend ? » qui conduit le chercheur à prêter attention au cas. En ayant en tête le postulat que ce qui est produit pas un sujet est pour lui une conduite ou une parole cohérente, « psycho-logique », on peut dire que la clinique ne recherche pas La Vérité, mais une vérité, celle du sujet interviewé » (Ménecier, 2018, p. 48).

Giust-Desprairies (2006, p. 168) précise que par cette rencontre « la clinique procède de la co-construction de sens qui se reprend dans les ajustements entre explications et associations du ou des sujet(s) et écoute et propositions interprétatives du clinicien ». Le savoir construit par la clinique est donc co-construit, en écho à la co-pensée, de Widlöcher (2013). II nomme ainsi l'élaboration conjointe issue de la rencontre analysant-analyste dans 


\section{eJRIEPS 49 avril 2021}

la cure psychanalytique. Avec précaution (puisque la demande est inversée et le cadre social autre), ceci peut être extrapolé à la rencontre sujet-chercheur. En effet un entretien de recherche est également fondé par la parole entre deux sujets (et donc soumis au phénomène transférentiel et à ses effets) qui cherchent ensemble à en comprendre davantage sur l'un d'entre eux en utilisant la parole comme outil. Une recherche clinique désigne donc « un espace où une pratique trouve à se théoriser en partant d'une situation singulière et de l'implication de celui qui y est compris comme professionnel » (Cifali, 1991, p.5). À partir de la rencontre (ici fortuite, car liée au contexte d'une visite pédagogique en classe) entre un sujet et un chercheur s'allume chez ce dernier un désir de comprendre ce qu'il en est de la cause de la parole ou de la conduite du sujet. Cette cause est à comprendre comme la " raison vraie ", soit comme le fondement inconscient de ce qui anime la conduite d'un sujet. On peut avancer que quand un sujet parle de ce qui le pousse à agir ou à être ou à dire comme il le fait, se télescopent dans son discours trois catégories de causes. Les pseudos raisons (surmoïques) " on m'a obligé, j’ai été forcé... ", les bonnes raisons (moïques) « j'aime cela, je souhaite me transformer en faisant/disant cela, je veux faire plaisir à... », et des « raisons vraies » qui relèvent de l'inconscient (du Ça) sur lesquelles le sujet a le plus souvent une quasi impossibilité à poser des mots. Ce dernier type de raisons renvoie à la vérité lacanienne, soit ce qui cause le sujet, le désir qui le pousse à agir.

Le désir de comprendre du chercheur, écho à sa pulsion épistémophilique (le désir de savoir freudien) induit deux attitudes. D'abord la disponibilité à ce qui surprend dans ce qu'il constate, variante de l'attention flottante freudienne qui demande d'attendre sans attente. Ensuite la disposition (d'esprit et d'habileté) à bricoler un lien entre ce que dit le sujet et ce qui s'entend dans sa parole.

Le chercheur clinicien se doit en effet d'être le bricoleur décrit par Levi Strauss (1962). Ce dernier explique que le bricoleur est apte à s'arranger avec les moyens du bord et ne sait pas exactement à l'avance quelle forme aura le résultat de son labeur. Cela revient pour le chercheur, lors des entretiens, à savoir-y-faire avec l'ensemble des paroles proposées par le sujet et plus particulièrement avec ce qui lui saute aux oreilles (ce qui fait effet de signifiant en deçà la signification). Lorsque quelque chose s'écoute dans ce qui est dit, c'est parce que les mots, comme les objets utilisés par le bricoleur, sont réutilisés avec une autre fonction que celle qui leur était attribuée initialement (dans la grammaire et la syntaxe pour la parole) et qu'ils sont réagencés dans une autre construction lexicale. Une fois mis en lien avec les outils conceptuels et les éléments théoriques de la psychanalyse, les mots « signifiantisés » (dotés d'une valeur affective, le quantum d'affect freudien) permettent de 


\section{eJRIEPS 49 avril 2021}

faire quelque chose de différent de la parole entendue, quelque chose de différent du sens commun, de l'énoncé. Quelque chose qui enseigne sur la structure psychique de celui qui parle.

L'ensemble des moyens et des procédures du clinicien bricoleur n'est donc pas définissable par un projet/objectif établi totalement à l'avance comme chez le chercheur ingénieur qui a planifié hypothèse/protocole/analyse/résultats. Une recherche clinique en sciences de l'éducation s'ancre ainsi dans une logique exploratoire et pas confirmatoire. II ne s'agit pas d'essayer de montrer qu'une intuition ou une hypothèse sont justes, mais de s'efforcer de comprendre la cause et la nature d'un fait observé, entendu dans la réalité d'un moment d'éducation ou de transmission.

\subsection{Les entretiens comme recueil de données}

Les entretiens de recherche clinique (ERC) utilisés dans ce travail sont cousins des «entretiens clinique de recherche» (Moro,1993) et des « entretiens non directifs de recherche » définis par Yelnick (2005). Quelques éléments néanmoins singularisent les ERC.

Si un entretien est le fait de discuter avec au moins une personne sur un thème défini par un des deux locuteurs, il faut garder à l'esprit qu'en latin dis-cutere signifie « secouer, frapper pour faire tomber ». II y a donc dans un entretien de recherche quelque chose d'inconnu qui peut tomber à l'issue de la discussion. Cette rencontre en parole repose sur l'acceptation sans condition du propos du sujet entendu (fut-il surprenant ou illogique) afin d'aider la production par ce sujet d'un discours continu et structuré sur et autour du thème abordé.

Le chercheur, animé d'une attitude accueillante (qui n'exprime ni ne montre de jugement de valeur sur ce qu'il entend), aide un sujet à dire quelque chose, plus qu'il ne pense en savoir, sur ce qu'il traverse. Ce sujet, à travers une parole élaborative guidée, fait en effet surgir à ses oreilles et à celles du chercheur le sens qu'il donne à la situation qu'il vit ou aux conduites qu'il adopte. Ce qui est dit l'est parfois pour la première fois. Rappeler en effet que le principe d'un ERC est de faire émerger « l'impensé de la problématisation de départ » (Vial, 2005, p.2) chez le sujet à qui on donne la parole. Le sujet et le chercheur deviennent alors des partenaires de production de savoir tout au long des différents entretiens. Comme le rappelle Savournin (2014), le présupposé, dans ce type de relation est que « le savoir est du côté du sujet » et non du côté du chercheur. Il y a ainsi « une bascule à opérer de la part du chercheur ». Cette inversion épistémologique demande et provoque une transformation 


\section{eJRIEPS 49 avril 2021}

du rapport au savoir (Beillerot, 1989) de chacun. Les deux sujets de l'ERC sont en effet les producteurs co-dépendants d'un savoir, non seulement le temps que dure leurs entretiens, mais également dans l'après-coup. Des années après chez le sujet (ce que montre Loizon, 2015) ou lors de l'écriture des résultats (ou les entretiens écrits continuent de représenter la voix du sujet) chez le chercheur.

L'ERC se base sur la logique des « entretiens non directifs » (Blanchet, 1991), mais même dans cette disposition, et contrairement à certaines idées reçues, « un entretien n'est jamais neutre. Ce n'est pas un simple recueil de données »(Blanchet, 1991, p.110). Cela dit, l'impact du chercheur, choisissant la non-directivité, se minimise par des attitudes et questions/relances orientant le moins possible la parole de l'autre. Pas de questions fermées ou confirmatoires avec introduction de signifiant nouveau (du type journalistique qui demande par exemple : «est-ce que vous vous sentez en sécurité ? ». Cette formulation oblige celui qui est questionné à répondre oui (ou non) en reprenant le substantif (sécurité) dans sa parole, alors que de lui-même il ne l'aurait peut-être pas choisi pour illustrer son sentiment).

Dans son attitude d'écoute, le chercheur clinicien se met dans la posture d'attention flottante freudienne, « un peu comme s'il se trouvait près d'une rivière, les doigts dans le courant, légèrement écartés pour laisser passer l'eau en filtrant ce qui pourrait arriver de l'amont, en l'occurrence des mots qui feront réagir son oreille » (Grégorcik, 2018, p. 57). II s'agit alors de se décentrer de ce qui se dit pour se pencher vers ce qui s'entend. Autrement dit le chercheur ne réagit pas comme dans une conversation, ni dans son écoute, ni dans son discours.

Un ERC engage le chercheur à commencer la rencontre par une question large ("Que pourrais-tu me dire sur le combat ? Qu'en penses-tu ?) puis de se contenter de relancer la parole du sujet en demandant des compléments de temps, de lieux, d'acteurs ("Les questions, que je t'ai entendue poser à Mme $D$, à qui d'autre les poses-tu? »), des illustrations ("Tu as dit "j'aime bien l'EPS", peux-tu me donner un exemple de ce que tu as bien aimé récemment en EPS ?»), pour chercher s'il existe une unité structurelle chez le sujet (et en rendre compte). De plus, quand le chercheur entend un «mot gros », soit un signifiant qui prête à l'équivoque dans sa propre structure signifiante ou qui, dans une discussion serait important et dont l'acception irait d'elle-même (" c'est une bonne prof "), il se doit de ne pas le recevoir sans questionnement et de demander des précisions (Assia : « c'est une bonne prof... », le chercheur "Ah ? C'est-à-dire ? Peux-tu illustrer? »). Dans ce moment de discussion du signifié (le sens lexical d'un mot), apparaît souvent la teneur 


\section{eJRIEPS 49 avril 2021}

signifiante que le mot prend dans l'architecture psychique du sujet. Sa valeur affective se déploie (comme on ouvre une carte routière) alors souvent de façon surprenante et riche (Assia : «Bonne prof? Ça veut dire qu'elle te force pas à parler »).

Reste que la conversation peut s'éloigner de ce qui fonde l'objet de l'ERC et que le sujet peut passer/rester dans la logorrhée d'un «bla-bla » de défense ou de résistance, « apparemment superficiel » (Gilbert, 2007, p. 282), sans intérêt étiologique avéré.

Pour éviter l'écueil du flou ou de la dérive de la conversation, il est possible de guider davantage la parole du sujet, c'est le postulat d'un entretien semi-directif. Alors, la conversation n'est ni totalement ouverte ni guidée par des questions précises. Le chercheur conçoit des questions-guides relativement ouvertes avant l'entretien, mais s'attache à respecter l'ordre du discours de la personne interrogée. Il s'efforcera simplement de recentrer l'entretien sur «ses » questions (centrées par l'objet de recherche) chaque fois que le sujet entendu s'en écartera. Mais cette technique induit un biais puisque les questions du chercheur introduisent souvent des signifiants nouveaux et que l'on assiste à la domination langagière et imaginaire du chercheur.

Pour faire avec ces deux écueils, les ERC menés dans ce travail ont donc été des entretiens que l'on peut nommer « semis non directifs “. En écho symétrique aux entretiens cliniques « semis directifs menés de façon à tendre vers la non-directivité » présentés par Gilbert (2007, p.280), ils gardent la trame de l'échange au gré de la parole du sujet, comme dans des entretiens non directifs, mais intègrent l'idée de questions « balises » de l'entretien semi-directif. Cependant si ces questions élaborées en amont de l'entretien sont écrites par le chercheur, et que ce dernier espère des réponses, elles ne sont jamais posées explicitement lors de la conversation.

Celles de ce travail étaient : «Que penses-tu du fait que tu demandes des choses sur toi à Mme D? », 'Pourquoi (et pour-quoi, cause et but) demandes-tu ces choses sur toi à Mme $D$ ?, «Qu'est-ce que tu espères qu'elle te réponde ? », «Est-ce que tu poses ce type de questions à tes parents? À d'autres enseignants ?».

Avoir ces questions écrites devant soi pour ne pas les perdre « de vue » au cours de l'entretien, étaye une stratégie de l'écoute productrice de significations (Blanchet \& Gotman, 1992) et rejoint ce que Paillé \& Mucchielli (2003) appellent un référent interprétatif initial. Comme elles ne sont pas dites et que le chercheur reste centré sur ce que dit le sujet, sur le dire qui peut s'entendre dans ce dit, et sur l'éclaircissement de ce dit semble, ces questions non posées ne sont que des aides à 'la sélection, l'inférence, la comparaison [des mots entendus] par rapport aux objectifs de l'entretien' (Blanchet et Gotman,1992, p.72). 


\section{eJRIEPS 49 avril 2021}

II s'agit en fait d'une tentative d'éthique de l'entretien ou le chercheur met à plat ses attentes informulées pour essayer de les considérer sans qu'elles influencent trop directement la rencontre (dans une logique contre transférentielle) et l'interprétation qu'il ne manque pas de se faire de ce qu'il entend.

\subsection{L'interprétation et l'implication du chercheur}

«La clinique psychanalytique est née du remaniement provoqué par la découverte de l'inconscient freudien dans la clinique psychiatrique » (Savournin, 2014). À l'observation des comportements s'est substituée l'écoute de ce que le sujet peut en dire, considérant que sa parole, aussi originale soit-elle, est sérieuse. Reste que la parole du sujet en soi n'est pas « une donnée » puisque pour prendre sa mesure de dévoilement du psychisme de celui qui parle, elle doit être interprétée par le chercheur qui écoute (puis qui lit les retranscriptions). L'interprétation est une forme « d'analyse du discours » (Maingueneau, 1996 ; KerbratOrecchioni, 1980)) qui s'efforce de repérer et de commenter les effets de subjectivité involontairement véhiculée par une parole adressée ». Interpréter un propos selon cette orientation consiste en un écumage (au sens culinaire du mot) des entretiens pour attraper, dans la parole du sujet, l'écart entre signifiant et signifié (le son et le sens), les récurrences, l'équivocité des mots, les blancs, les trébuchements, soit tout ce que Devereux (1967, p. 201) nomme les « auto-trahisons involontaires ». Elles permettent de dévoiler une partie de la vérité du sujet (comme le font les lapsus dans la vie courante).

Interpréter les dits du sujet demande aussi de percevoir « les voix multiples dans sa parole », soit d'entrevoir la place imaginaire qu'il prend et celle à laquelle il met le chercheur quand il s'exprime. Autrement dit comment fait-il avec le « qui tu es pour moi, qui je suis pour toi » pointé par Benveniste (1966) ?

L'acte d'interpréter repose sur le fait que ce qu'entend le chercheur (le sens latent) peut être autre chose que ce qui a été dit ou que le sujet a voulu/cru dire et/ou faire entendre (le sens manifeste). C'est dans cet écart entre le dit et l'entendu, reposant sur les effets de métaphore et de métonymie, que se meut la subjectivité du chercheur en tant qu'instrument de recherche. Ce moment de la recherche repose sur les « insight » du chercheur. L'insight, contraction de la locution anglaise « inner sight» (le regard intérieur), désigne la solution lumineuse (Marková, 2009) qui naît dans les réflexions du chercheur. Cette construction cognitive et subjective, arrivant presque par surprise à la conscience, permet de comprendre la dynamique du cas, d'attribuer une cause à ce qui est entendu ou observé et de relier ce qu'il présente avec des connaissances ou des concepts. En effet, écouter la parole de l'autre 


\section{eJRIEPS 49 avril 2021}

de façon interprétative, autrement dit sélective, revient au final à donner une signification au discours du sujet à l'aune des éléments théoriques du champ psychanalytique.

La subjectivité, dans ces moments de la compréhension de l'autre, est un outil voire même « une qualité de l'analyse et non un biais à éviter » (Gilbert, 2007, p.286).

La clinique de ce travail demande donc aussi au chercheur d'analyser son poids dans le processus de recherche, de " s'y être repéré » dit Cifali (1994). Elle ajoute le chercheur « est responsable de sa subjectivité. Inutile de la cacher sous le masque d'une objectivité technique » (op. cit. p. ).

Mon implication est triple dans ce travail.

- Vis-à-vis de l'objet de recherche d'abord, car toute recherche clinique parle (aussi) du chercheur. Le désir qui m'anime dans les sciences de l'éducation passe souvent, depuis 15 ans, par de désir d'essayer de comprendre ce qui ne semble pas compréhensible dans une situation de transmission. Certains des mots d'Assia m'ont paru lors de la première écoute en classe incohérents par rapport à la situation sociale du cours que j'observais. Une élève de quatrième, habituellement, ne demande pas ce 'genre de chose, de cette façon "à son enseignante. C'est en prenant une parole comme le signifiant maitre d'Assia (le trait saillant, résumant sa structure) « madame dites-moi vous pour moi », mais sans en saisir l'origine ou le but, que j'ai voulu écouter son dit de façon interprétative. Dans cet « instant de voir » (Lacan, 1966b) je me suis demandé : « qu'est-ce que ça peut bien vouloir dire qu'Assia sollicite ça comme ça »?

- Vis-à-vis du processus de recherche ensuite (cf. l'ERC défini ci-dessus), soit du fait de la mise en jeu de ma subjectivité en tant que sujet observant, parlant et écoutant, lors des entretiens et interprétant lors de l'analyse de discours, dans l'après coup lors des « insight » formulant des causes explicatives (le temps pour comprendre, Lacan, 1966b) et de la rédaction des résultats et conclusions (le moment de conclure, Lacan, 1966b).

Dans ces trois temps, c'est bien 'l'authenticité avec [ma] singularité radicale qui permet d'interpréter l'autre' (Labridy, 2012, p.13)

- Vis-à-vis de l'effet du chercheur. Levi Strauss (1955) a établi en quoi la présence du chercheur étrangère au sujet ou à l'objet a des effets sur les actes et les paroles du sujet rencontré (observé et entendu). Dans le cas présenté, je ne suis pas intervenu sur les conduites d'Assia pendant cours, m'efforçant de me faire le plus discret possible. Néanmoins, je suis venu l'observer une fois dans un cadre officiel accompagné de deux autres visiteurs puis une seconde fois seul. Cette présence ne peut pas ne pas avoir été remarquée par Assia. On ne peut gommer « l'immixtion involontaire du chercheur » (De 


\section{eJRIEPS 49 avril 2021}

Landsheere, 1969) dans l'occasion sociale qu'il analyse, et même assis, au bord du gymnase, j'ai eu un impact sur les élèves et leur professeure. Devereux (1967, p.223) suggère de reconsidérer la question des rapports entre sujet observateur et sujet observé en s'inspirant du modèle liant patient et analyste dans la cure psychanalytique. II soutient que « le principe méthodologique classique qui commande au chercheur de tout mettre en œuvre pour considérer ce qu'il observe d'un point de vue strictement objectif est non seulement vain, mais surtout contre-productif ». II ajoute (op. cit. p.30) 'par bonheur, ce qu'on appelle les « perturbations » dues à l'existence de l'observateur, lorsqu'elles sont correctement exploitées, sont les pierres angulaires d'une science du comportement authentiquement scientifique et non - comme on le croit couramment - un fâcheux contretemps dont la meilleure façon de se débarrasser est de l'escamoter'.

Les demandes d'Assia auraient-elles été aussi nombreuses, et de cette teneur, sans ma présence étrangère? Freud (1905) a montré que c'est parce qu'il y a un troisième auditeur que bien des plaisanteries sont émises en public, notamment que des grivoiseries sont dites à une femme. Ce qui est dit s'adresse à la femme présente, mais est énoncé pour être entendu par un spectateur extérieur au duo. En sachant qu'un autre adulte était là, Assia n'a-t-elle pas davantage demandé à son enseignante ? Même si Mme D confesse que « c'est vrai qu'Assia me demande beaucoup. Mais elle sait qu'elle peut me demander », on peut aussi penser qu'Assia a questionné en sachant que sa professeure se sentait sommée de répondre, par une sorte de pression surmoïque imaginaire (devoir se monter aux yeux du visiteur comme la professeure qui sait répondre, qui ne laisse pas sans réponse).

\subsection{L'étude de cas}

\subsubsection{La construction de cas comme analyse des données et présentation des résultats}

Une recherche orientée par la psychanalyse fait souvent référence à l'étude de cas, ne serait-ce que parce que « l'aboutissement d'une investigation clinique, c'est l'histoire d'un cas » (Lagache, 1949, p. 166). Comment dire mieux à propos de cette méthode que Revault d'Allones et Giami (1989, p.16) : «L'étude de cas vise non seulement à donner une description d'une personne, de sa situation et de ses problèmes, mais elle cherche aussi à en éclairer l'origine et le développement, l'anamnèse ayant pour objet de repérer les causes et la genèse de ces problèmes ». 


\section{eJRIEPS 49 avril 2021}

Cette façon de construire et de présenter un savoir, aux terminologiques multiples (« leçon publique à la Salpêtrière », «présentation de malade à Sainte Anne », « vignette clinique » (Huguier \& Maisonneuve, 1990)), repose sur la présentation d'une singularité que doit rendre intelligible la théorie. II s'agit de donc de s'employer à « rapporter un événement à son contexte et à le considérer sous cet aspect pour voir comment il s'y manifeste et s'y développe » (Hamel,1997, p.10). En d'autres mots, il s'agit de saisir comment un contexte scolaire (EPS en APSA combat, activité physique d'opposition mélangeant lutte et judo fréquemment pratiqué en EPS) donne lieu chez un sujet à des questions incongrues. Dans un champ épistémologique différent on pourrait dire que le cas permet de considérer un fait d'éducation comme un événement situé (Leplat, 2012).

Le cas doit, aussi et surtout, déborder la théorie et ne pas en être une illustration. II ne s'agit pas de penser que toutes les adolescentes vivant le combat en EPS vont se conduire comme Assia, vis-à-vis de leur professeure. II s'agit juste de penser le cas Assia comme un élément supplémentaire pour renseigner ce qui peut se jouer pour un sujet pris dans l'APSA combat en présence d'une professeure. Cette perspective est le contraire du désir d'universaliser le cas, devenant alors catégorie. Le 'travail d'écriture [qui] doit rendre vivant le portrait du sujet' (Vial, 2005, p.3) est tenu de relever les signes généraux sous-jacents à la singularité exposée. Singularité qui, dans ce travail, n'est pas réductible à celui d'un exemplaire de l'adolescence ou de la relation éducative.

Le cas cherche à mettre en lumière des processus qui rendent un sujet unique tout en soulignant son côté que Cifali (2007) qualifie de "profondément humain ». C'est de cette universalité « profondément humaine » que naît la scientificité et donc la légitimité, de la recherche par construction de cas unique.

La recevabilité du cas unique comme outil de production de connaissances a été mise en évidence par Flyvberg (2006). Accepter la scientificité de l'étude de cas demande au fond d'y voir une autre conception du savoir ; un savoir qui ne peut prétendre à la complétude ou à la totalité et qui fait que « la construction d'un cas s'appuie sur une logique du pas-tout. Celle-ci indique qu'il manquera toujours quelque chose, qu'il n'y a pas de vérité absolue de l'expérience. » (Bonnaud, 2013, p. 153).

C'est pour cela que ce sont des hypothèses interprétatives qui sont proposées en guise de résultats. Le mot hypothèse pour signifier que parmi l'ensemble des pistes explicatives possibles issues de ce que les paroles analysées suggèrent, ce sont les plus plausibles (au regard du lien paroles des sujets/interprétation/corpus de connaissances théoriques) qui sont écrites. Si elles sont justes pour la théorie, elles ne prétendent pas à une valeur de 


\section{eJRIEPS 49 avril 2021}

vérité. Le chercheur les pose comme des interprétations possibles, mais pas certaines. En effet il n'existe pas d'interprétation finale, car le sens ne s'épuise jamais, il y a toujours un sens suivant. La possibilité d'interprétation de ce qui est dit n'est jamais finie, car aucune énonciation ne se réduit à son énoncé.

Le cas proposé ici est donc que Fédida (1995) nomme une écriture du figurable à propos de ce que peut traverser une adolescente qui pratique une ASPA, sur sa parole adressée à sa professeure et sur une façon de faire professorale avec ce dire.

\subsubsection{Présentation d'Assia}

Assia est une élève de $4^{\mathrm{e}}$ dans un collège ZEP du nord de Paris. Elle a « 13 ans $1 / 2$ ». Je l'ai observée, en EPS dans l'APSA combat en EPS lors de la visite d'une professeure stagiaire. Le but de cette observation n'était pas « clinique », mais pédagogique et je l'effectuais en tant que formateur INSPE. Néanmoins, comme souvent en clinicien «à plein temps » (l'expression est de Loureaux, 1976), j'ai été surpris par certaines questions d'Assia (vers sa professeure), étrange dans leur forme et leur récurrence. « Madame, ma jambe ditesmoi vous !», " Madame, comment je peux savoir si je suis comme il faut? ", " J'arrive pas à voir si je suis bien mise », "Je vois pas comment il faut que je sois, madame, s'il vous plaît... »

J'ai pris ce que Mme D sa professeur appelle « ses drôles de questions », comme une demande, au sens psychanalytique du terme. Le sens commun fait de la demande une énonciation adressée à un autre avec l'attente d'une réponse. Ce que la psychanalyse propose de supplémentaire à la notion de la demande, c'est sa différence avec la notion de besoin. « Si la demande peut porter au départ sur un objet, elle reste insatisfaite et tributaire de la dynamique désirante du sujet qui parle et qui s'adresse à autrui » précise Crommelinck-de Henau (2001, p. 176).

Fort de cet adossement théorique, j'ai souhaité en savoir plus sur la signification et la cause de ces énonciations surprenantes. Je suis retourné observer Assia de façon clinique dans la leçon d'EPS suivante (sans attente précise, mais désireux de repérer une cause à ses questions), et ai constitué un verbatim de ses paroles (le verbatim est une prise de notes manuscrite, mot pour mot, de certaines paroles dites par les sujets, mais qui ne reprend pas la totalité du discours entendu). Certaines de ses demandes lors de ce second cours ont conforté le lien particulier qu'elle avait établi avec son enseignante «C'est mon bras ou son bras qu'est là ? », " Je vois pas comment il faut que je sois moi... », " Madame, pouvez me 


\section{eJRIEPS 49 avril 2021}

dire si ça va parce que moi j’ai réussi... ", "C'est comme ça que vous voulez que je sois mise ? »

À la fin de cette seconde visite d'observation, j'ai demandé à Assia si elle accepterait de me rencontrer pour parler d'EPS. Je lui ai précisé que j'étais "professeur de professeur" et que mon travail consistait à comprendre ce qui se passait pendant les cours d'EPS entre les professeurs et les élèves et que je voulais mieux comprendre sa relation avec Mme D Je lui ai signalé que les entretiens seraient enregistrés puis retranscrits par écrit, mais que son anonymat serait garanti de façon à ce que ni son nom, ni le nom de sa professeure, ni le nom du collège ne soient mentionnés.

Après qu'elle ait accepté, j'ai contacté le chef d'établissement et les parents d'Assia et ai obtenu leur accord. Nous nous sommes donc rencontrés deux fois au CDI du collège (un troisième rendez-vous initialement prévu n'a pas été possible).

J'ai ensuite réalisé avec elle deux ECR "semi-non directifs" enregistrés (45 et 55 minutes). J'ai également, après la seconde visite eu une conservation de 45 minutes avec Mme D dont j'ai établi le verbatim.

\section{4- Le cas Assia, résultats et discussion}

\subsection{Une demande provoquée par une transformation du corps exacerbée en EPS par l'APSA}

Toute parole étant à resituer dans l'histoire du sujet, on peut entendre que les "drôles de questions" d'Assia sont prises entre des causes structurelles (les métamorphoses adolescentes que vit son corps et sa structure psychique) et contextuelles (le rapport au corps imposé par l'APSA combat).

Assia explique ses transformations adolescentes : "depuis cette année, je mesure un peu plus d'un $1 \mathrm{~m} 60$ et 47 kilos, ma mère me dit que j'ai grandi trop vite". Elle ajoute, "c'est vrai que maintenant je m'habille plus en 14 ans, mais en taille adulte, en taille M". "Comme ma mère en fait.", précise-t-elle. Dans cette parole il est possible d'entendre la proximité entre la taille médium et l'initiale $\mathrm{M}$ de "mère", sa référence d'adulte féminin à laquelle elle s'identifie et qu'elle aime/M. Pour signaler sa division entre enfance et âge adulte elle ajoute, "mais j'ai que 13 ans 1/2". Ses turbulences intimes semblent suractivées, amplifiées par ce que l'APSA combat demande à son corps "J'avais jamais fait avant et c'est pour ça que je comprends pas trop comment tu dois faire, y'à l'autre qui t'écrase, ... elle m'attrape partout". L'écart entre je qui ne comprend pas et tu qui doit faire, au-delà de la formule langagière, 


\section{eJRIEPS 49 avril 2021}

signale peut-être quelque chose de la division imaginaire et symbolique provoquée chez Assia par les formes de corps demandées dans les immobilisations en combat et par le rapport si proche avec l'adversaire. Elle explique très clairement le poids de la logique corporelle et d'interaction motrice induite par cette activité, car, quand je lui demande si elle questionne aussi Mme D dans l'APSA vécue en parallèle, pendant l'autre leçon d'EPS, elle dit : « non, non, en natation, je vois mes jambes, je les contrôle bien. En fait tu vois tes jambes donc ça va. En combat tu te vois pas tes jambes...en combat je préfère que ce soit elle qui me dise pour moi ».

Dans cette APSA combat, le contact avec le corps de l'autre oblige à avoir des sensations tactiles inhabituelles. L'opposition et la proximité font en sorte que des parties du corps (le ventre, les jambes, le dos, la poitrine, les bras) qui n'ont pas cet office habituellement deviennent des récepteurs tactiles privilégiés (par la pression, la saisie de l'autre). Cette nouvelle géographie sensorielle permet d'être renseigné sur son corps par des indications extéroceptives issues d'endroits coutumiers d'informations intéroceptives. Cela dit, ce qu'elle ne voit pas « [ses] jambes », elle demande à l'autre de les voir pour elle.

Les paroles d'Assia permettent de penser qu'elle demande en fait d'en savoir plus sur elle, au-delà de son corps « capacitaire » (Andrieu et Paintendre, 2015). Elle souhaite recevoir de l'autre un réassurage sur ce qu'elle vit et sur la limite entre corps perçu et corps vécu modifiée par les nouvelles expériences physiques du combat. On peut penser que dans ce moment Assia est en proie à une confusion entre la perception de son schéma corporel (Shilder, 1935), soit la représentation qu'elle peut se faire consciemment de son corps et son image inconsciente du corps (Nasio, 2002). Le combat semble pour Assia augmenter le sentiment d'avoir un corps « habit d'Arlequin » (Wallon, 1959), patchwork de sensations, de représentations et de pouvoirs plus ou moins assemblés et ajustés. Le regard et la voix de Mme D jouent un rôle de réunificateurs.

\subsection{Une demande révélatrice du transfert}

On peut entendre dans les paroles d'Assia (leur contenu et leur forme), la trace d'une relation spéciale avec son enseignante, et plus précisément une concrétisation du transfert freudien.

Toute relation sociale dissymétrique entre deux sujets (professeur-élève, médecin-malade) engage dans un phénomène d'identification que Freud (1945) a nommé le transfert. Le transfert définit dans la cure la projection sur la personne de l'analyste de sentiments existant à l'état latent chez le patient. La position inégale entre analyste et analysant 


\section{eJRIEPS 49 avril 2021}

résonne comme une trace du positionnement infantile (enfant-parent) ou l'un « sans savoir " (voire sans parole) attend de l'autre des réponses sur ce qu'il vit et ressent. C'est le repérage et le maniement de cette disposition psychique qui permet à l'analyste d'aider le patient (en posant des mots dessus) à transformer son rapport inconscient avec « le quelque chose » (Réel) vécu dans le passé qui l'embarrasse ou le traumatise dans le présent sans qu'il ne puisse en dire mot.

Il est admis que la situation d'enseignement est propice au transfert (Ferenczi, 1908 ; Pfister, 1924 ; Zulliger, 1930 ; Aichorn, 1925; Filloux, 1974, l'ont montré). Parce qu'un(e) enseignant(e) peut devenir une figure isomorphe aux parents pour certains élèves, la relation éducative est organisée par la reviviscence chez ces derniers de désirs, d'affects, éprouvés envers les parents dans la prime enfance et adressés cette fois à un nouvel objet de désir : le professeur.

Cifali (1994, p. 127) évoque le transfert dans ces termes : « le rapport à un autre qui pousse à revivre ce qu'on ne peut prononcer tout seul ». Ponnou (2014, p. 75) précise quant à lui : « le sujet se lie à l'Autre du langage en lui supposant un savoir ». Lacan (2001a) a précisé en effet que celui qui est objet de transfert est considéré par l'autre comme « sujet supposé savoir ». Cette relation tient au fait que l'être humain cherche toujours des réponses à ce qu'il rencontre et qui l'embarrasse dans sa vie ; qu'il espère toujours en savoir plus sur lui, sur ce qu'il est et sur ce qu'il éprouve. Dans le cas contraire, il suppose toujours qu'il y a une personne qui possède la réponse aux questions de sa vie.

Les questions surprenantes d'Assia : «Madame, dites-moi si je suis bien !?» semblent bien dévoiler une teneur transférentielle au lien qu'elle tisse avec Mme D ?

Assia le confirme en disant : «Mme $D$, elle sait nous voir, elle... Elle sait mieux nous dire que nous si on est juste ou pas ». Cette relation est d'autant plus forte qu'Assia expose comment ses parents sont manquants du côté de cette aide à sa construction imaginaire. Quand je lui demandais si elle exprime ce genre de chose à ses parents, Assia explique : « bon, je répète les prises chez moi avec ma sœur... mais mes parents, non je ne leur demande rien sur moi, ils sauraient pas. Mon père il m'enverrait chier, excusez-moi du terme quoi, il parle pas de ça. Et ma mère? Elle me dirait ma fille t'es assez grande, débrouille-toi avec tes jambes et tes prises (rires) ». Assia, ainsi, sur le mode angoissant, fait l'expérience du manque dans l'autre qui « ne parle pas de Ça (freudien) » et engage à se débrouiller avec un corps grandissant pas encore compris ou assumé chez Assia. Sans conflit de loyauté, elle assume de choisir Mme D, pour en savoir plus sur elle, Mme D qui « elle, répond toujours, elle sait bien nous dire ». Noter l'équivoque de cette formule qui ne 


\section{eJRIEPS 49 avril 2021}

permet pas d'entendre si Assia veut dire «bien dire à nous ou bien dire ce que nous sommes ».

En d'autres termes, Assia fort d'un effet de transfert, semble utiliser Mme D pour construire son image du corps. Cette notion renvoie à l'idée (la représentation plus la sensation), en perpétuel remaniement, que chacun se fait de son corps. Elle traduit ce que l'être humain perçoit à chaque moment et dans la relation aux autres des qualités de son corps (image et possibilités physiques). L'image du corps est liée à l'histoire personnelle (affective, psychologique, physiologique). Selon Sanglade (1983, p. 106) «l'image du corps peut également être assimilée à la représentation de soi. Cette représentation de soi dépend des relations aux autres et de leur qualité. Elle peut s'éprouver solide ou détruite, désirée ou rejetée, elle est liée à l'épreuve du narcissisme et à la vie relationnelle ».

Pour résumer le lien particulier qui semble unir Assia et Mme D, Lacan (1973b, p. 133) précise ; " dès qu'il y a quelque part le sujet supposé savoir, il y a transfert ». Le contexte particulier de l'école fait qu'un professeur est placé par un élève en « Sujet supposé savoir » quand celui-ci attend de lui une/des indication(s) sur quelque chose de qu'il est, sur son être singulier et intime, une réponse aux questions existentielles qui le taraudent sans qu'il puisse forcément les formuler. Un élève n'attend pas dans cette relation ce qu'il escompte quand il place « le professeur comme "sujet supposé savant" (connaissant les programmes) ou "sujet supposé sachant" (l'aider à apprendre et à grandir) » (Montagne, 2021). C'est bien une relation transférentielle qui semble établie entre Assia et Mme D.

Reste que, fort du postulat de Lacan (1972, p. 1), « toute question ne se fonde jamais que sur une réponse. On ne se pose de questions que toujours là où on a déjà une réponse », il est possible d'avancer qu'Assia interprète ce qu'elle est en train de vivre en combat. C'est justement parce que cette interprétation sur le hors sens de ce qu'elle ressent lui apporte des réponses « personnelles » qui l'insécurisent, ou l'insatisfont, qu'elle demande l'avis de l'Autre (Mme D). Elle attend en quelque sorte un retour de mise en mot (du Symbolique) sur son éprouvé (du Réel) faute de se pouvoir elle-même se voir (Imaginaire). La psychanalyse souligne bien l'importance du fait que c'est l'assentiment de l'autre référent qui donne recevabilité à ce qui n'est qu'une intuition logique d'identification (entre lui et l'image qu'il perçoit de lui) du sujet.

La relation transférentielle s'est dévoilée davantage quand, lors du second entretien, le discours d'Assia s'est orienté autour du signifiant "bonne prof” ». Assia précise ; “Une bonne prof' ??? Elle sait te dire sur toi ! Par exemple, c'est Mme D, par exemple, vous avez vu qu'elle écoute, qu'elle nous respecte quand on lui demande, elle répond toujours. Et 


\section{eJRIEPS 49 avril 2021}

quand tu fais un truc, elle dit quand ça veut dire ça ou ça, elle te dit... elle nous facilite à nous comprendre pour mettre notre corps bien et tout ça, quoi... Elle nous regarde souvent, elle est assez proche, mais des fois aussi, elle te laisse tranquille... Ça veut dire qu'elle te force pas à parler ».

On peut penser que dans ce cours d'EPS, Mme D sait « dire sur toi », elle « te dit " (dire à toi ou dire sur toi ?), et arrive à traduire en mots les sensations de corps et « dit quand ça veut dire ça ou ça ». La similitude avec la mère d'Assia, "qui a 43 ans comme Mme D ", mais qui lui ne lui offre que son non savoir en disant, "débrouille toi avec tes jambes", participe du processus de projection d'Assia sur Mme D, qui " sait mieux me voir que moi. Je sais pas vous expliquer en vérité, mais je sais qu'elle saura me dire pour moi ». II semble bien, que dans sa façon d'être professeur face à cette élève Mme D, qui " te laisse tranquille», mais reste "assez proche», recréée quelque chose d'un lien parental manquant.

Paradoxalement, Mme D dit ne pas avoir conscience de ce positionnement signifiant : "Mon attitude? Non, je suis normale, je suis moi, le suis là quoi ». J'ai entendu " je suis la quoi ...(silence) ", au-delà d'une formule langagière, comme une énonciation du savoir manquant dont se sert Mme $\mathrm{D}$, comme une question à trou posée au chercheur. Interrogation témoignant de l'effet du transfert à un autre niveau, entre Mme D et moi.

\subsection{Une demande poussée par la rencontre adolescence/sport de combat, rappelant quelque chose du stade du miroir: "Madame, dites-moi vous ! Comment je suis? "}

Dans la conduite d'Assia, reliant sensations de corps/parole de l'Adulte/regard de l'Adulte, se lit quelque chose qui renvoie au stade du miroir (Lacan, 1966a ; Wallon, 1934). Cette étape de la construction de l'être humain, entre 6 et 18 mois, désigne le fait que l'enfant dans les premiers temps de sa vie ne perçoit pas son corps comme une totalité unifiée. II n'arrive pas à distinguer son corps et les sensations qu'il procure de ce qui lui est extérieur. II le perçoit comme quelque chose de dispersé, de morcelé. Lors de la confrontation à son image, face à un miroir, en compagnie d'un adulte (la mère) qui nomme l'image que l'enfant voit de lui dans le miroir. Alors, «l'image de lui, les sensations ressentis et son nom se fusionnent pour créer ce que Freud a nommé le moi » (Nasio, 2002, p.83). Par ce moment l'infans (l'enfant qui ne parle pas) reconnaît sa propre image parce qu'elle est nommée. Dès lors l'enfant ne s'identifie plus seulement à ses sensations, mais au l'image de lui « dans un miroir » et à la parole de l'autre qui nomme cette image et donc le 


\section{eJRIEPS 49 avril 2021}

nomme. Dans cette phase de structuration corpo-psychique le lien à l'autre est donc double, il y a l'autre qui voit et l'autre qui nomme.

Assia en demandant, « Madame, pouvez me dire si mon bras est là aussi parce que moi j'ai réussi... C'est mon bras ou son bras qu'est là ?... Ma jambe elle est où, s'il vous plaît ? ", avoue qu'elle ne sait plus si ce qu'elle ressent du positionnement des parties de son corps sont conformes à ce qu'elle en connait. Elle avoue que son schéma corporel et son image du corps sont perturbés et qu'elle en a une lecture peu précise.

Reste que l'image de soi aperçue dans le miroir n'est pas complète (le sujet est plus que l'image de lui) ni exacte (le sujet est autre chose que l'image qu'il a ou donne de lui). Le stade du miroir entraîne donc paradoxalement une réunification du sujet, mais aussi une division fondamentale de son être. Le déchirement de l'unité imaginaire est un leurre qui vole d'autant plus en éclat à l'adolescence. Les transformations intimes du corps, le changement de l'image de soi dans le regard des autres font que le sujet « adolescent » est divisé entre ce qu'il ressent, ce qu'il pensait être, et l'image qu'il donne de lui aux autres (et à de lui-même dans son miroir).

Bouleversée par les changements adolescents de son corps (qui a « grandit trop vite») exacerbés par les sollicitations corporelles et kinesthésiques de l'APSA combat, Assia semble prise dans ce que Freud (1923) appelle un défaut de traduction et demande à Mme $\mathrm{D}$, « comment je peux savoir si je suis comme il faut?».

Le corps et les événements de corps provoqués par l'adolescence et ses remous, deviennent alors inaccessibles au langage ou aux représentations. Assia dans cette double contrainte corporelle semble prise dans « processus de transformation d'énergie, de symbolisation et de signification »(Green, 1976, p. 126 ). Elle témoigne d'une sorte d'inquiétude (inquiétude interne) consécutive au fait de ne pouvoir ni ressentir ni se représenter, ni dire son corps changé et sur-sollicité par le corps de l'autre, l'adversaire du combat. En disant « Je vois pas comment il faut que je sois... ", elle en appelle à Mme D pour lui redire comment elle doit être pour ressembler à l'image idéale qu'elle se fait d'elle. Elle illustre ainsi le constat de Nasio (2014, p. 210) : «Que je me distingue de l'autre ou que je me sente semblable à lui, que je me sente autonome ou que je dépende de lui, j'ai toujours besoin de l'autre pour être moi"

Ce que vit Assia en combat renvoie bien (en partie, puisqu'Assia parle) à ce que vit le nourrisson du stade du miroir, soit l'impuissance motrice et posturale, "l'incoordination des fonctions, la discordance des pulsions" (Wallon, 1934, p. 223). Le parallèle entre la nature et la cause des demandes d'Assia repose sur le fait que, si pour Wallon, le stade du miroir 


\section{eJRIEPS 49 avril 2021}

est une étape dans la chronogenèse de l'être humain destinée à être vécue puis dépassée, pour Lacan (1966a) elle est une forme de relation à l'autre qui reste prégnante toute sa vie durant. Relation présente spécialement quand le sujet se trouve en situation de "désaide", soit de solitude face à l'affrontement d'une situation angoissante ; le combat en EPS pour Assia.

\subsection{Une demande illustrant le rôle de l'autre dans la perception que le sujet a de lui}

La psychanalyse soutient que la structuration du sujet, et donc sa compréhension, ne peut être que "socio-logique", que l'être humain se construit avec, par, parfois contre, l'autre ; l'autrui social.

Pour formaliser le rôle du regard et de la parole de l'autre dans cette structuration du sujet, Lacan propose le schéma L (1978, p. 53) (cf. figure 1). Cette formalisation montre que l'être humain (le sujet Assia), pour se percevoir en passe par les autres (petit a) qui lui renvoient, par une relation imaginaire (axe1), une image de lui (image d'Assia). Ce lien se superpose, s'oppose, se complémente, avec le lien de parole qui unit le sujet à l'Autre (grand A) et le constitue sur le plan symbolique (axe2). Le grand Autre (A majuscule) en opposition avec le petit autre (a minuscule, qui représente autrui, le semblable) est dans la théorie lacanienne l'ensemble de ce qui est extérieur au sujet et plus particulièrement l'ordre symbolique (du langage) qui le détermine. 


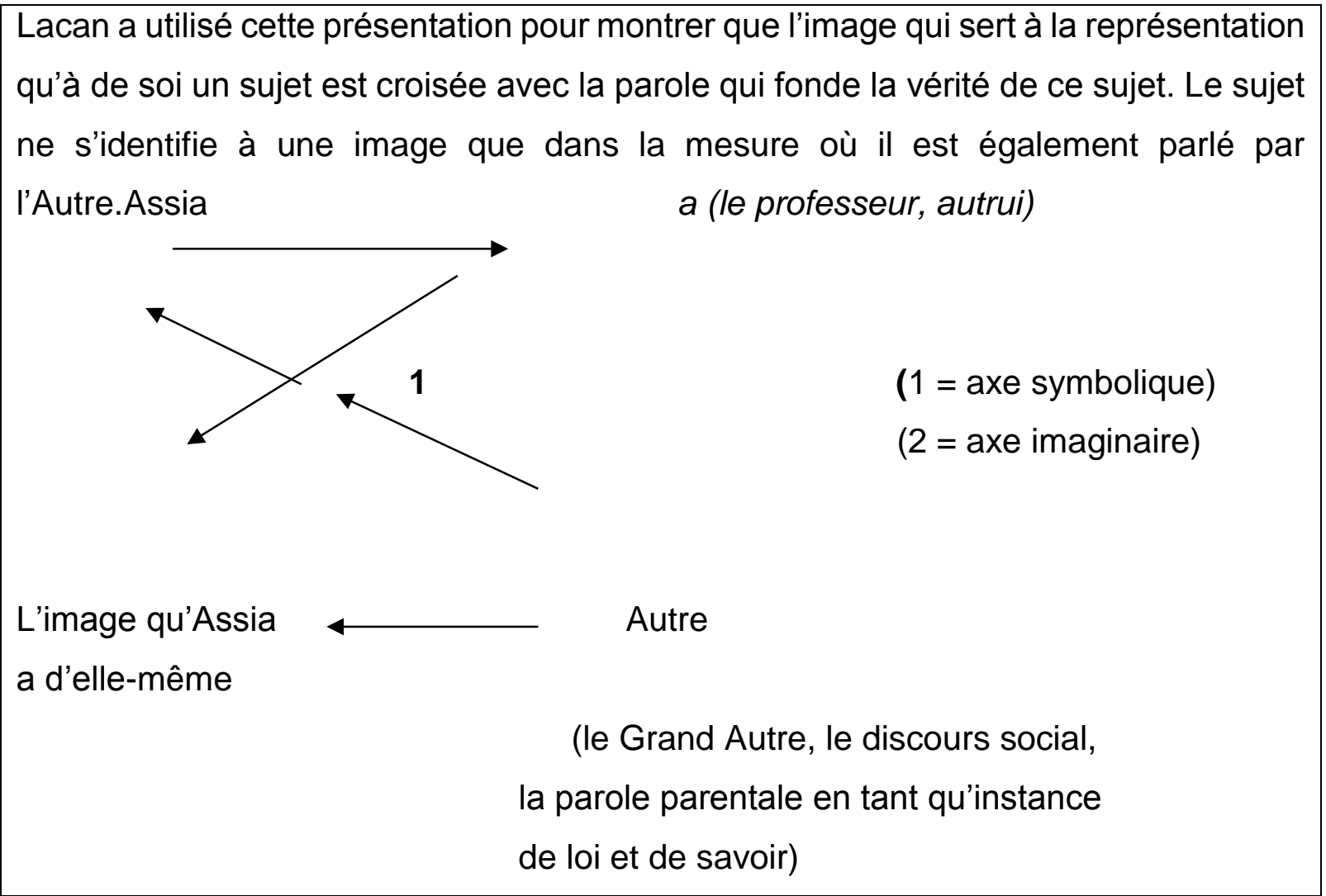

Figure 1 : Le schéma $L$ appliqué à Assia et Mme D

Se montre ici le fait que le sujet ne peut trouver son unité avec lui-même qu'à partir de la relation à son semblable et ne peut prendre conscience de lui que dans le processus de reconnaissance portée par le langage. Le désir de cette reconnaissance semble pouvoir être une cause des demandes d'Assia.

4.5. La réponse de Mme $\mathrm{D}$, « une bonne prof ", qui " répond pas vraiment »; une autre façon d'être professeur en EPS

Les observations de leçons, comme les entretiens avec Assia et avec Mme D, montrent que les réponses de $M m e D$ sont satisfaisantes pour Assia. La nature de ces réponses permet de comprendre que c'est la position subjective de cette professeure, plus le contenu pédagogique ou didactique de ses réparties, qui offre à Assia une réponse à sa question " comment et qui suis-je ? ».

En premier lieu, noter que la position désirante de Mme D, qui dit «demandez moi » aux élèves, montre qu'elle les imagine comme capables de demander, qu'elle ne les réifie pas, mais les considère comme des "parlêtres" ('être humain parlant lacanien). L'équivoque de sa formule «demandez-moi » (demander Mme D ou demander « à » Mme D ?) a fait 


\section{eJRIEPS 49 avril 2021}

office de signifiant pour Assia; Mme D est celle qui a des réponses. En ne précisant pas ce qu'elle attendait comme type de demande, Mme D a laissé la parole entr'ouverte à plus que du savoir ou des conseils «EPS».

On peut avancer que cette proposition a-signifiante (sans substantif, mais pas sans signification) a en partie a provoqué le transfert d'Assia. S'illustre ici le postulat de Žižek, (2011) selon lequel le transfert du sujet dans la cure est relatif au fait que l'analyste croit dans les capacités du patient à en dire sur lui plus qu'il ne pense en savoir sur lui. N'est-ce quelque chose de nature comparable qui se joue ici ? N'est-ce pas en premier lieu parce que Mme D a fait comprendre à Assia (et aux autres élèves) quelle était capable de « la/lui » demander, que les demandes arrivent ? Par cela Mme D s'est établie en « sujet supposé croire » que ses élèves allaient demander. Elle a considéré ses élèves comme sujets supposés désirer/demander. Lacan (1998) précise, « une parole n'est parole que dans la mesure exacte où quelqu'un y croit ».

Quand je lui ai demandé si elle avait perçu l'attitude d'Assia, Mme D m'a dit : « c'est vrai qu'elle me demande beaucoup, je la laisse dire ». C'est ici que semble se loger « le savoiry faire » de Mme D (X, 2013). Elle a perçu que les questions d'Assia étaient une demande et, qu'à ce titre, elles devaient être dite, accueillies, qu'elles n'attendaient pas de réponse explicite dans l'énoncé. En d'autres termes qu'elle ne devaient pas recouvrir le désir d'Assia par ses propres mots, soit ne pas proposer d'Aïode (la parole qui donne le savoir).

Mme D précise : «Assia voit que je l'entends, je la regarde, mais je ne sais pas si je lui réponds. Je la regarde et je fais oui, comme ça (elle hoche la tête affirmativement) sans parler, je réponds, mais pas vraiment ».

Cette réponse singulière, (Assia verrait une écoute...) est une réponse vide, mais signifiante. En effet cette «pas vraiment» réponse ne veut pas dire absence de réponse. Mme $D$, ne répond à Assia ni du côté didactique ni du côté pédagogique, mais répond en regardant, en assurant le point de regard, sans mots, en se contentant par moment de hocher la tête de façon affirmative. Avec toutes les précautions éthiques et épistémologiques possibles dédouanant d'un copier-coller réducteur, on peut penser que cette réponse contient des éléments sémiotiques et signifiants de nature voisine de celle qu'un analyste peut avoir dans une cure. Quand le patient demande « qu'est-ce que c'est que ça qui me satisfait, ou m'angoisse ?», l'Autre, analyste ne lui répond pas par la parole, mais par sa présence silencieuse. En lui montrant ainsi la limite de son savoir sur «ça », et ne jouant pas le rôle qu'on lui suppose, il lui permet de passer à une autre étape du rapport que le sujet entretient avec son symptôme. Bien entendu cette façon de répondre sans 


\section{eJRIEPS 49 avril 2021}

réponse ne vaut pas pour tous et toujours. Ce n'est pas une technique ou une compétence langagière, c'est une façon singulière d'incarner un discours qui aide sans trop dire. En fait Mme D structure son lien avec Assia depuis le discours de l'Analyste (Lacan, 2001b). Discours (Op’s) qui dit oui (qui accueille), qui entend au-delà du dit, qui questionne sans parole et ne répond pas seulement à l'énoncé, mais aussi à l'énonciation.

Assia le signale d'une autre façon quand je lui demandais si Mme D était une bonne professeure : “Ma prof' c'est quelqu'un qui te force pas à parler sans arrêt. Un bon prof' comme Mme D c'est plutôt un attendeur, enfin vous voyez quelqu'un qui attend quoi, plutôt qu'un interrogateur, qui t'oblige à parler ». Dans cette phrase se dessine une autre façon d'endosser la posture (au sens de Bucheton et Soulé, 2009) d'enseignant d'EPS face à un adolescent : Etre «attendeur».

\section{Perspectives : Des moments de parole authentique en formation des enseignants}

Expérimenter la saisie d'une demande dans une question, les effets du transfert, le lien au sujet supposé savoir, se confronter à un formateur «attendeur », sont des éléments que vivent les enseignants participants à un Groupe de Parole et d'Analyse de Pratique (Montagne, 2018). Ces moments de formation où se créée une inversion de la demande (ce sont les participants qui savent et pas le formateur) et la bascule dans le rapport au savoir, évoquée supra (Savournin, 2014), permettent vraiment de changer la compréhension et la relation que les enseignants ont avec la parole. Ils éprouvent le fait de dire quelque chose que l'on ne savait pas savoir ou d'écouter ce qui ne se dit pas. Les GPAP permettent aussi d'accueillir la demande d'être entendu et de diluer le sentiment d'être pris entre infantilisation et abandon que manifeste souvent les enseignants stagiaires. Ils regrettent qu'on leur impose ce qu'ils doivent faire autant qu'ils déplorent d'être laissés sans aide effective. Grâce à cette formation, ils pourront expérimenter qu'un formateur ne « réponde pas vraiment » et peut-être en être satisfait.

On peut penser de la sorte former les enseignants à faire la classe et donner cours en lâchant un peu de la position de maîtrise toute, qui voudrait qu'un enseignant soit toujours dans « le discours du Maître (qui commande) et de l'Université (qui sait) » (Lacan, 2001b) et qu'il doive toujours répondre. Mme D montre qu'une compétence pédagogique langagière peut être de répondre «pas vraiment ». Assia témoigne qu'une bonne prof peut « ne pas forcer à parler». II est question ici de penser autrement les compétences 7 et P2 du référentiel des compétences des enseignants de 2015 : « Maîtriser la langue française ». Elle peut « ne pas seulement demander de parler bien pour servir de modèle linguistique 


\section{eJRIEPS 49 avril 2021}

"top-down" ou de jouer au correcteur de syntaxe " (Montagne, 2020). Maîtriser la langue, c'est aussi écouter ce qui se dit dans ce qui s'entend, répondre à la demande et pas seulement aux questions, faire en sorte que les élèves soient demandeurs, bref montrer une compétence " éthique » de respect de l'autre. Compétence que Cifalli (1994) nomme un savoir-faire d'altérité responsable (dans le sens de répondre de, mais aussi de répondre à). L'omniprésence de la parole, et des échanges verbaux permanents entre professeur et élèves en EPS, rend cette perspective presque incontournable.

Au-delà de cet enrichissement personnel et professionnel, ces moments de rencontre en parole permettent aux enseignants d'analyser ce qui se passe (ou pas) dans leurs classes et de questionner « l'effet professeur » (Montagne, 2017, p. 267) soit «l'impact subjectif du lien d'inconscient à inconscient entre un professeur et un élève sur le rapport au savoir et l'engagement dans la réussite scolaire ». De tels moments donnent aussi l'occasion d'évoquer la place du corps dans la classe quand on enseigne à des adolescents. PujadeRenaud (1983) a montré il y a longtemps déjà les effets du corps en cours. L'EPS, bien entendu peut moins encore que les autres disciplines faire l'économie de cette prise en compte du rôle du trio " corps-parole-regard » dans le lien éducatif. Mais n'est-ce parce qu'un nombre certain d'enseignants d'EPS partagent le savoir-y-faire langagier de Mme D que les élèves aiment aussi nombreux l'EPS ?

De plus, la pratique et l'étude des GPAP montre que s'engager dans une analyse en parole de leur pratique permet aux enseignants, dans l'après coup, de davantage s'apercevoir comment ils font en réalité avec leurs élèves. Lors de notre entretien, Mme D a fait le constat de l'effet que sa propre parole adressée peut avoir sur le sujet qui parle : " c'est vrai que quand je vous dis ça je m’aperçois que je lui réponds pas... mais ça lui va, alors je vais sûrement continuer (rires) ».

\section{Pour conclure}

Ce travail s'attachait à comprendre, en EPS, la cause psychique et la nature de paroles d'élèves surprenantes et à identifier la nature de la réponse d'une professeure et les bénéfices que tirait cette élève de ces réponses.

L'analyse interprétative des paroles d'Assia et de Mme D permet d'avancer que ces questions sont causées par le croisement des modifications de l'adolescence, l'APSA combat et la rencontre avec une professeure écoutante. Cette dernière, à son insu, permet que se rejoue pour cette élève quelque chose du stade du miroir dans ses cours d'EPS. Cette relation la renseignant autant sur son corps que sur ce qu'elle est comme sujet. 


\section{eJRIEPS 49 avril 2021}

Les questions d'Assia sont à prendre comme une demande (dans le symbolique) de savoir (dans l'imaginaire) sur ce qu'elle est et vit (dans le Réel). Elle demande à en savoir sur son être et pas seulement sur des compétences techniques et motrices. Ses questions surprenantes sont l'expression d'une demande de « dire-secours » (Lacan, 2006) pour en savoir plus sur elle grâce à l'autre.

Les «pas vraiment » réponses de Mme D, émises depuis le discours de l'Analyste (1970) sont telles qu'elles offrent en quelque sorte un feed-back scopique et oral qui fait dire à Assia, dans une formule une fois encore équivoque : "Mme D est une prof avec qui je m'entends bien ". On peut voir dans cette formule un angle de perception de la relation pédagogique supplémentaire et fécond pour l'EPS : la parole de cette professeur décerne (au sens d'attribuer) en quelque sorte un corps à cette adolescente.

Les paroles d'Assia et de Mme $D$ témoignent ainsi du fait que l'adulte à un rôle fondamental dans la corporéïsation et l'unification moïque de l'adolescent, « non seulement impuissant face aux nombreuses transformations corporelles dont il fait l'objet, mais sommé de comprendre, seul, ce qu'il en est » (Montagne \& Bui-Xuan, 2015, p. 7).

L'APSA combat en EPS, dans sa logique interne et ce qu'elle impose de confrontation au corps fait qu'une " unhiemlichkeit » (l'incroyable étrangeté freudienne) peut surgir chez un sujet (un élève) et faire que ce dernier ait besoin du regard et de la parole d'un autre sujet (le professeur) pour conforter ce qu'il est vraiment.

L'économie pulsionnelle provoquée par l'EPS (le lien corps/satisfaction) n'est pas pour tous les élèves une source de satisfaction. En fonction des circonvolutions psychiques singulières, il peut en résulter un « Unlust » (déplaisir pouvant aller jusqu'à la souffrance). Ce terme freudien témoigne de l'augmentation de tension interne à un organisme. Par l'analyse clinique interprétative des dires d'Assia se dessine une autre lecture de l'effet EPS sur le processus adolescent et une autre façon de tenir la position professorale. "Je suis obligée de demander à la prof, pour voir, quoi... ", dit Assia. Elle résume de la sorte que, dans un gymnase, à l'image du " pubertaire savant » (Gutton, 2007), le sujet peut inventer comment faire avec son embarras, mais pas sans autre. Dans d'impossibilité de réaliser "son selfie subjectif », voire même de ne pas savoir quoi voir ("pour voir quoi ? ») Assia en appelle à Mme D, pour photographier et légender ce qu'elle vit. Anna Freud (1945) a réfuté le fait qu'un enfant puisse percevoir sa souffrance clairement et formuler une demande explicite sur ce qu'il ressent comme peut le faire un adulte. On peut penser, fort des paroles d'Assia et de la teneur de sa relation avec Mme D, que cette limitation touche aussi un adolescent 


\section{eJRIEPS 49 avril 2021}

Ce constat va à l'encontre de l'idée reçue en l'EPS qui avance que " de nature " les adolescents auraient besoin d'autonomie, et que cette norme est bonne pour tous. Ce postulat (qui a par ailleurs des vertus), issu de l'agrégat « Piaget + méthodes actives » est en partie infondé en tant que norme structurelle. II renvoie au fait que les adolescents sont victimes des projections des adultes à l'école. Si beaucoup, d'adolescents veulent une « parentectomie », au sens Doltosien, certains, comme Assia, ne veulent pas être sans autre. Cela demande de reconsidérer avec intérêt et attention la « magistrale dérobade » que Méard \& Bertone (1998) signalaient déjà il y a plus de vingt ans dans un autre paradigme scientifique.

Le cas «Assia et Mme D » souligne que, pour peu que l'enseignant permette aux élèves de parler (et pas seulement de verbaliser) et ose écouter « en vérité » ce qui se dit dans ce que les élèves disent, les bénéfices de l'EPS peuvent espérer dépasser les seules compétences scolaires et réellement participer à la réussite éducative de tou-te-s.

Une autre enseignante me disait il y a peu à propos de ses élèves, « ils sont inouïs ». La signification du mot, inouï « qui n'a jamais été entendu », dresse bien l'état et la perception du sujet adolescent à l'école. Assia, elle, donne de la voix pour se faire ouïr, et Mme D qui a l'oreille (et la parole) fine lui répond. Elles posent un contre point au constat de Huerre(1997, p. 43), « les adolescents nous cherchent, le problème, c'est qu'ils ne nous trouvent pas ». Mme D en bon « attendeur », a su se faire trouver.

Fort de l'avertissement de Dor (1985, p. 181) : "Si la demande est avant tout expression du désir, elle est immédiatement double. Par-delà la demande de satisfaction du besoin se profile la demande de « l'en plus » qui est avant tout demande d'amour", une autre posture de formateur/professeur compétent dans l'usage de l'agapé (l'amour de l'autre) se dessine alors. S'offrir à la demande, entendre, puis attendre et répondre, « mais pas vraiment ».

\section{Bibliographie}

Andrieu, B. \& Paintendre, A. (2015). Le corps capacitaire des adolescent(e)s : une émersion du vivant dans leur perception du step. Revue Staps, 108, 49- 59.

Arrivé, M. (2003). Langage et inconscient chez Freud: représentations de mots et représentations de choses. Cliniques méditerranéennes, 68, 7-21.

Aichorn, A. (1925). Jeunesse à l'abandon. Toulouse : Privat, 1973.

Beillerot, J. (1989). Le rapport au savoir : une notion en formation. In Beillerot, J., Bouillet, A., Blanchard-Laville, C. \& Mosconi, N., Savoir et rapport au savoir : élaborations théoriques et cliniques (pp. 165-202). Paris : Éditions Universitaires. 


\section{eJRIEPS 49 avril 2021}

Bentata, H. (2011). La Voix de Sirène D'une incarnation mythique de la voix maternelle. Essaim, 26, 63-73.

Blanchard-Laville, C. (2001). Les enseignants entre plaisir et souffrance. Paris : PUF. Blanchet, A. (1991). Dire et Faire Dire : l'Entretien. Paris : Armand Colin.

Blanchet, A. \& Gotman, A. (1992). L'enquête et ses méthodes : l'entretien. Paris : Nathan. Bonnaud, H. (2013). L'inconscient de l'enfant. Paris : Navarin.

Bonnaud, H. (2015). Le corps pris au mot. Paris : Navarin.

Bucheton, D. \& Soulé, Y. (2009). Les gestes professionnels et le jeu des postures de l'enseignant dans la classe : un multi-agenda de préoccupations enchâssées. Éducation \& didactique, 3, 29- 48.

Buznic -Bourgeacq, P. \& Terrisse, A. (2013). Le sujet en position d'enseignant : pratiques et discours de trois professeurs d'EPS débutants avec ou sans expérience personnelle dans l'activité enseignée. Revue française de pédagogie, 184, 15-28.

Carnus, M.-F. (2010). L'inscription clinique en didactique de l'éducation physique et sportive. Cliopsy, 4, 73-88.

Cifali, M. (1994). Le lien éducatif : contre-jour psychanalytique. Paris : PUF.

Cifali, M. (1991). Modèle clinique de formation professionnelle, apports des sciences humaines, théorisation d'une pratique. Communication orale, in Séminaire IUFM, « Du métier d'enseignant au parcours de formation ». Marseille, 1-3 juillet 1991.

Cifali, M. (2002). Travail de notre subjectivité pour une dignité de nos actes. In (Coord.) A. Piquenot, II fait moins noir quand quelqu'un parle (pp. 157-167). Dijon : CRDP Bourgogne. Cifali, M. (2017). Action poétique dans le monde de la science et de la formation. In $G$. Amado, J.-P. Bouilloud, D Lhuilier \& A.-L. Ulmann, La créativité au travail (pp. 107-126). Paris : Eres.

Crommelinck-de Henau, B. (2001). Au-delà du manifeste : la cause voilée de la demande de l'enfant. Cahiers de psychologie clinique, 16, 175- 190.

Devereux G, (1967). De l'angoisse à la méthode dans les sciences du comportement. Paris : Payot, 1998.

Dor, J. (1985). Introduction à la lecture de Lacan. Vol. 1. Paris : Denoël.

Fédida, P. (1995). Le site de l'étranger. La situation psychanalytique. Paris : PUF.

Ferenczi, S. (1908). Psychanalyse et pédagogie, in, Psychanalyse I, CEuvres complètes, 1908-1912. (pp. 51-67). Paris : Payot, 1994.

Filloux, J. (1974). Du contrat pédagogique. Paris : L'Harmattan, 1996. 


\section{eJRIEPS 49 avril 2021}

Flyvbjerg, B. (2006). Five Misunderstandings about Case-Study. Research. Qualitative inquiry, vol. 12, 2, 219-245.

Freud A. (1945). Le Traitement psychanalytique des enfants. Paris : PUF, 1981.

Freud, S. (1895). Esquisse d'une psychologie. Paris : Eres, 2011.

Freud, S. (1905). Le mots d'esprit et sa relation à l'inconscient. Paris : Gallimard, 1992.

Freud, S. (1915). Pulsions et destins des pulsions. Paris : Payot, 2012.

Freud, S. (1923). Le moi et le ça. In Essais de psychanalyse (pp. 177-234). Paris : Payot, 1981.

Gilbert, S. (2007). La recherche qualitative d'orientation psychanalytique : l'exemple de l'itinérance des jeunes adultes. Recherches qualitatives, hors-série 3, 274-286.

Gregorcik, V. (2018). Les enseignants et l'enseignement explicite : entre ignorance et résistance. Une étude clinique orientée par la psychanalyse, Mémoire de Master Ingénierie de La recherche en Éducation non publié. INSPE Besançon, Université de Franche Comté. Green, A. (1976). Le discours vivant: La conception psychanalytique de l'affect. Paris : PUF, 2002.

Giust-Desprairies, F. (2003). La clinique : enjeux d'une position épistémologique. In M. Cifali \& F. Giust-Desprairies (Dir). De la clinique; Un engagement pour la formation et la recherche (pp. 165-186). Bruxelles : De Boeck.

Gutton, P. (2007). Le pubertaire savant. Adolescence, 60, 347-358.

Hamel, J. (1997). Étude de cas et sciences sociales. Paris : L'Harmattan.

Huerre, P., Pagan-Reymond, M. \& Reymond, J-M. (1997). L'adolescence n'existe pas.

Paris : Odile Jacob.

Huguier, M. \& Maisonneuve H. (1990). La rédaction médicale. De la thèse à l'article original. La communication orale. Paris : Doin, 1990.

Kerbrat-Orecchioni C. (1980). L'énonciation de la subjectivité dans le langage. Paris: Armand Colin.

Labridy, F. (1997). Dire la performance. In Brousse, M.-H., Labridy, F., Terrisse, A. \& Sauret, M.-J. Sport, Psychanalyse et Science (pp. 65-75). Paris : PUF.

Labridy, F. (2012). Des gadgets qui nous font courir. In, A. Chottin, Y. Grasser, F Labridy, Y-C Stavy. Le regard et la voix chez l'enfant. Incidences clinique (pp. 7-19). Lille : ACF CAPA.

Lacan, J. (1966a). Le stade du miroir comme formateur de la fonction du Je, telle qu'elle nous est révélée dans l'expérience psychanalytique. In Les Écrits (pp. 93-101). Paris : Seuil. 


\section{eJRIEPS 49 avril 2021}

Lacan, J. (1966b). Le temps logique et l'assertion de certitude anticipée. Un nouveau sophisme. In Les Écrits (pp.197-213). Paris : Seuil.

Lacan, J. (1972). Conférence de Louvain. Texte établi par Jacques-Alain Miller, La Cause du Désir, 2017/2, 96, 7-30.

Lacan, J. (1973b). Les quatre concepts fondamentaux de la psychanalyse, Séminaire $X$, 1963-1964. Paris : Seuil.

Lacan, J. (1975). Le Séminaire1974-1975, Livre XXII, RSI, (Réel, Symbolique, Imaginaire), Inédit.

Lacan, J. (1978). Le Séminaire1954-1955, Livre II, Le moi dans la théorie de Freud et dans la technique de la psychanalyse. Paris : Seuil.

Lacan, J. (1998). Le séminaire, Livre I, Les écrits techniques de Freud, 1953-154. Paris: Seuil.

Lacan, J. (2001a). Le Séminaire, Livre VIII, Le transfert, 1960-1961. Paris : Seuil.

Lacan, J. (2001b). Radiophonie. In Autres écrits (pp. 403-446). Paris : Seuil.

Lacan, J. (2006). Le Séminaire, Livre XVI, D’un Autre à l'autre, 1968-1969. Paris : Seuil.

Lagache, D. (1949). Psychologie clinique et méthode clinique. L'évolution psychiatrique, 1, 155-178.

Landsheere, (De), G. (1969). Comment les maîtres enseignent. Analyse des interactions verbales en classe. Bruxelles : Ministère de l'Éducation nationale.

Leplat, J. (2012). De l'étude de cas à l'analyse de l'activité. Perspectives interdisciplinaires sur le travail et la santé [En ligne], 4-2 | 2002, mis en ligne le 23 septembre 2012, consulté le 6 mars 2020.

Lévêque, M. (1985). Contribution à une lecture différentielle des pratiques sportives. Enfance, 38/4, 403-418.

Lévi-Strauss, C. (1962). La Pensée sauvage. Paris : Gallimard, 2008.

Loizon, D. (2015). Intervenir en EPS pour développer l'entretien de soi. Une étude de cas d'un enseignant expérimenté qui débute. Carrefours de l'éducation, 40, 51 - 65.

Loureaux, R. (1976). Sociologue à plein temps. Paris : L’Épi.

Maingueneau, D. (1996). Les termes clés de l'analyse du discours. Paris : Seuil.

Marková, I.-S. (2009). L'insight en psychiatrie. Paris : Doin.

Méard, J. \& Bertone S. (1998). Le professeur d'EPS et les attitudes d'élèves. Dossier Revue EPS 38. Paris : Ed Revue EPS. 


\section{eJRIEPS 49 avril 2021}

Ménecier, L. (2018). Alchimie enseignante: Analyse orientée par la psychanalyse d'une efficacité professorale, Mémoire de Master Ingénierie de la Recherche en Éducation. INSPE Besançon, Université de Franche Comté non publié.

Moll, J. (2003). Des effets du regard et de la parole. Enfances \& Psy, 24, 50-56.

Montagne, Y.-F. (2014). O estádio do inspelho em Educação Física : localizar o traço pulsional nas perguntas de um aluno para um estudo de caso, Estilos da clinica, 19 (3), 482-498.

Montagne, Y-F \& Bui-Xuan, G. (2015). Adolescence, une transhumance subjective. Revue STAPS, 108, 7-11.

Montagne, YF. (2017). Refuser d'entrer dans les pratiques physiques en EPS. Regard psychanalytique sur trois études de cas. In (Coord.) G. Cogérino, Rapport au corps, genre, réussite en EPS (pp. 259-274). Paris : AFRAPS.

Montagne, YF. (2018). Formateur d'enseignants, enseignant-chercheur clinicien, animateur de Groupes de Parole et d'Analyse de Pratique, tamponnages subjectifs et façons de faire. Revue Travail et Apprentissages, 18, 87-109.

Montagne, YF. (2020). Introduction. In Y-F. Montagne (Coord.), La parole (pp. 7-11). Paris : Revue EPS.

Montagne, YF. (2021). Sujet, Savoir et supposés, le regard de la psychanalyse. In (Coord) E. Brossais \& M.-C. Carnus. Savoir(s) et Sujet(s) dans la formation aux métiers de l'enseignement, de l'éducation et de la formation. Bordeaux : PUF.

Moro, M.-R. (1993). Les méthodes cliniques. In. R. Ghiglione \& JR., Richard. Cours de psychologie. Bases, méthodes, épistémologie (pp. 461-507). Paris : Dunod.

Nasio, J.-D. (2002). Mon corps et ses images. Paris : Payot.

Paillé, P. \& Mucchielli, A. (2003). L'analyse qualitative en sciences humaines et sociales.

Paris : Armand Collin.

Pfister, O. (1924). Die Psychoanalytische Methode, Leipzig : Kleinkhardt.

Ponnou, S. (2014). Lacan et l'éducation. Paris : L'Harmattan.

Postic, M. (1979). La relation pédagogique. Paris : PUF.

Pujade-Renaud, C. (1983). Le corps de l'élève dans la classe. Paris : ESF.

Revault d'Allonnes, C. \& Giami, A. (1989). La démarche clinique en sciences humaines. Paris : Dunod.

Sami Ali, M. (1990). Corps réel, corps imaginaire. Paris : Dunod.

Sanglade, A. (1983). Image du corps et image de soi au Rorschach. Psychologie française, t. 28-2, 104-111. 


\section{eJRIEPS 49 avril 2021}

Savournin, F. (2014). Le rapport à l'épreuve. In Colloque Rapport au savoir d'enseignants en formation : Approche clinique d'orientation psychanalytique, 3-4 octobre 2014, Campus Walferdange, Luxembourg, non publié.

Schilder, P. (1935). L'image du corps. Paris : Gallimard, 1968.

Soler, C. (2019). Retour sur la «fonction de la parole ». Paris : Ed Nouvelles du Champ lacanien.

Terrisse, A. (1997). La transmission du savoir dans une activité sportive : les sports de combat. In Brousse, M-H., Labridy, F., Terrisse A. \& Sauret. M-J., Sports, psychanalyse et science. (pp. 109-159). Paris : PUF.

Vial, M. (2005). Conférence au séminaire CREN, axe 3 Clinique de l'éducation et de la formation, Université de Nantes. ww.michelvial.com/Méthodologie clinique en sciences de l'éducation posture du chercheur, mise en ligne le 05/12/2005, consulté le 07 mars 2018. Wallon, H. (1934). Les origines du caractère chez l'enfant. Les préludes du sentiment de personnalité. Paris : PUF, 1983.

Wallon, H. (1959). Kinesthésie et image visuelle du corps propre. Enfance, 3-4, 252-263.

Widlöcher, D. (2013). Empathie et co-pensée. Journal de la psychanalyse de l'enfant, 3, 3944.

Yelnik, C. (2005). L'entretien clinique de recherche en sciences de l'éducation. Recherche \& Formation, 50, 133-146.

Žižek, S. (2001). Comment lire Lacan. Paris : Nous.

Zulliger, H. (1930). La psychanalyse à l'école. Paris : Flammarion, 1986. 